\title{
CONNECTION OF THE DIFFERENTIAL-GEOMETRICAL STRUCTURES WITH SKEW-SYMMETRIC DIFFERENTIAL FORMS. FORMING DIFFERENTIAL-GEOMETRICAL STRUCTURES AND MANIFOLDS
}

\author{
L.I. Petrova
}

Moscow State University, Russia, e-mail: ptr@cs.msu.su

The closure conditions of the inexact exterior differential form and dual form (an equality to zero of differentials of these forms) can be treated as a definition of some differential-geometrical structure. Such a connection discloses the properties and specific features of the differential-geometrical structures.

The using of skew-symmetric differential forms enables one to reveal a mechanism of forming differential-geometrical structures as well. To do this, it is necessary to consider the skew-symmetric differential forms whose basis, unlike to the case of exterior differential forms, are manifolds with unclosed metric forms. Such differential forms possess the evolutionary properties. They can generate closed differential forms, which correspond to the differential-geometrical structures.

\section{Connection between exterior differential forms and differential-geometrical structures}

In this section it will be shown a connection between exterior differential forms and some differential-geometrical structures. This connection allows to describe the properties and specific features of differential-geometrical structures.

The properties of differential-geometrical structures are based on the properties of closed exterior differential forms.

\section{Closed exterior differential forms}

The exterior differential form of degree $p$ ( $p$-form on the differentiable manifold) is called a closed one if its differential is equal to zero:

$$
d \theta^{p}=0
$$

(In more detail about skew-symmetric differential forms one can read in [1-3]. With the theory of exterior differential forms one can become familiar from the works $[4-8])$.

From condition (1) one can see that the closed form is a conservative quantity. 
The differential of the form is a closed form. That is,

$$
d d \omega=0
$$

where $\omega$ is an arbitrary exterior form.

The form, which is a differential of some other form:

$$
\theta^{p}=d \theta^{p-1}
$$

is called an exact form. Exact forms prove to be closed automatically [6]

$$
d \theta^{p}=d d \theta^{p-1}=0
$$

Here it is necessary to pay attention to the following points. In the above presented formulas it was implicitly assumed that the differential operator $d$ is a total one (that is, the operator $d$ acts everywhere in the vicinity of the point considered locally), and therefore, it acts on the manifold of the initial dimension $n$. However, a differential may be internal. Such a differential acts on some structure with a dimension being less than that of the initial manifold. The structure, on which the exterior differential form may become a closed inexact form, is a pseudostructure with respect to its metric properties. \{Cohomology, sections of cotangent bundles, the eikonal surfaces, the characteristical and potential surfaces, and so on may be regarded as examples of pseudostructures.\}

If the form is closed on pseudostructure only, the closure condition is written as

$$
d_{\pi} \theta^{p}=0
$$

And the pseudostructure $\pi$ is defined from the condition

$$
d_{\pi}^{*} \theta^{p}=0
$$

where ${ }^{*} \theta^{p}$ is the dual form. (For the properties of dual forms see [8]).

From conditions (5) and (6) one can see that the form closed on pseudostructure (a closed inexact form) is a conservative object, namely, this quantity conserves on pseudostructure.

\section{Differential-geometrical structures}

From the definition of a closed inexact exterior form one can see that to this form there correspond two conditions:

1) condition (5) is a closure condition of the exterior form itself, and

2 ) condition (6) is that of the dual form.

Conditions (5) and (6) can be regarded as a definition of some binary object that combines the pseudostructure and the exterior differential form being defined on this pseudostructure. Such a binary object can be named a BiStructure. The Bi-Structure combines both an algebraic object, namely, the closed exterior form, and a geometric one, namely, the pseudostructure as well. 
In its properties this differential-geometrical structure is a well-known GStructure. Here a new term Bi-Structure was introduced to distinguish it from G-structures to which there correspond closed inexact exterior forms.

The specific feature of this structure consists in the fact that it combines objects, which possess duality. The closed exterior differential form and the closed dual form are such objects. The existence of one object implies that the other one exists as well. It seems to be senseless to combine mathematically these two binary objects. However, this combined structure constitutes a unified whole that carries a double meaning. This statement will we explained later while considering a mechanism of forming the differential-geometrical structures.

From conditions (5) and (6) one can see that Bi-Stricture constitute a conservative object, namely, a quantity that is conservative on the pseudostructure. Hence, such an object (Bi-Structure) can correspond to some conservation law. (It is from such object that the physical fields and corresponding manifolds are formed.)

The connection of the differential-geometrical structures with exterior forms allows to understand the properties and specific features of the differentialgeometrical structures.

\section{The properties of exterior differential forms and differential-geometrical structures}

The basic properties of exterior differential forms are connected with the fact that any closed form is a differential.

The exact form is, by definition, a differential (see condition (3)). In this case the differential is total. The closed inexact form is a differential too. The closed inexact form is an interior (on pseudostructure) differential, that is

$$
\theta_{\pi}^{p}=d_{\pi} \theta^{p-1}
$$

And so, any closed form is a differential of the form of a lower degree: the total one $\theta^{p}=d \theta^{p-1}$ if the form is exact, or the interior one $\theta^{p}=d_{\pi} \theta^{p-1}$ on pseudostructure if the form is inexact. (This may have the physical meaning: the form of lower degree can correspond to the potential, and the closed form by itself can correspond to the potential force.)

\section{Invariant properties of closed exterior differential forms and differential-geometrical structures. Nondegenerate trans- formations}

Since the closed form is a differential, then it is evident that the closed form proves to be invariant under all transformations that conserve a differential. 
The examples of such nondegenerate transformations are unitary, tangent, canonical, and gradient transformations.

To the nondegenerate transformations there are assigned closed forms of given degree. To the unitary transformations it is assigned (0-form), to the tangent and canonical transformations it is assigned (1-form), to the gradient transformations it is assigned (2-form) and so on. It should be noted that these transformations are gauge transformations for spinor, scalar, vector, tensor (3form) fields. Hence one can see that the differential-geometrical Bi-Structure relate to the gauge type of the differential-geometrical G-Structure. They remain to be invariant under all transformations that conserve the differential.

The closure of exterior differential forms, and hence their invariance, results from the conjugacy of elements of exterior or dual forms.

From the definition of exterior differential form one can see that exterior differential forms have complex structure. Specific features of the exterior form structure are homogeneity with respect to the basis, skew-symmetry, integrating terms each including two objects of different nature (the algebraic nature for form coefficients, and the geometric nature for base components). Besides, the exterior form depends on the space dimension and on the manifold topology. The closure property of the exterior form means that any objects, namely, elements of the exterior form, components of elements, elements of the form differential, exterior and dual forms and others, turn out to be conjugated. (In the author's work [2] some types of conjugacy of exterior differential forms have been considered). A variety of objects of conjugacy leads to the fact that the closed forms can describe a great number of various differential-geometrical structures. In doing so, a connection of the differential-geometrical structures with exterior forms enables one to understand what the invariant properties of the differential-geometrical structures are conditioned by.

Since the conjugacy is a certain connection between two operators or mathematical objects, it is evident that, to express a conjugacy mathematically, it can be used relations. Just such relations constitute the basis of mathematical apparatus of the exterior differential forms. This is an identical relation. Identical relations of exterior differential forms disclose also the properties of differential-geometrical structures.

\section{Identical relations of exterior differential forms}

Identical relations of exterior differential forms reflect the closure conditions of differential forms, namely, vanishing the form differential (see formulas (1), (5), (6)) and hence the conditions connecting the forms of consequent degrees (see formulas $(3),(7))$. Since the closure conditions of differential forms and dual forms specify the differential-geometrical structures, identical relations for exterior differential forms specify the differential-geometrical structures too. (Here it 
should be noted that formulas (1) and (3) are not of interest for the differentialgeometrical structures).

The importance of the identical relations for exterior differential forms is manifested by the fact that practically in all branches of physics, mechanics, thermodynamics one faces such identical relations. The examples of such relations are the above presented Cauchy-Riemann conditions in the theory of complex variables, the transversality condition in the variational calculus, the canonical relations in Hamilton formalism, formulas by Newton, Leibnitz, Green, the integral relations by Stokes, Gauss-Ostrogradskii, the thermodynamic and characteristical relations, the Bianchi identities, the eikonal relations and so on. (In more detail about identical (and nonidentical) relations it is outlined in the author's work [3]).

The identical relations express the fact that each closed exterior form is a differential of some exterior form (with a degree less by one). In general form such an identical relation can be written as

$$
d_{\pi} \phi=\theta_{\pi}^{p}
$$

In this relation the form in the right-hand side has to be a closed one. (As it will be shown below, the identical relations are satisfied only on pseudostructures).

In identical relation (8) in one side it stands the closed form and in other side does a differential of some differential form of the less by one degree, which is a closed form as well.

The identical relations of another type are the analog of relation (8) obtained by differentiating or integrating this relation.

Identical relations of exterior differential forms are a mathematical expression of various types of conjugacy that leads to closed exterior forms. They describe a conjugacy of any objects: the form elements, components of each element, exterior and dual forms, exterior forms of various degrees, and others. The identical relations, which are connected with different types of conjugacy, elucidate invariant, structural and group properties of exterior forms that are of great importance in applications.

The functional significance of identical relations for exterior differential forms lies in the fact that they can describe a conjugacy of objects of different mathematical nature. This enables one to see internal connections between various branches of mathematics. Due to these possibilities the exterior differential forms, and correspondingly the differential-geometrical structures, have wide application in various branches of mathematics.

\section{Mechanism of forming the differential-geometrical structures}

It has been shown that the skew-symmetric closed exterior differential forms allow to describe the properties and specific features of the differential-geometrical 
structures.

In this section it will be shown that the skew-symmetric differential forms describe also a process of forming the differential-geometrical structures. However, to do this, one must use skew-symmetric differential forms, which, in contrast to exterior (skew-symmetric) differential forms, possess the evolutionary properties, and for this reason they were named evolutionary differential forms.

A peculiarity of the evolutionary differential forms consists in the fact that they generate exterior differential forms, which correspond to the differentialgeometrical structures. This elucidates a process of forming the differentialgeometrical structures.

A radical distinction between the evolutionary forms and the exterior ones consists in the fact that the exterior differential forms are defined on manifolds with closed metric forms, whereas the evolutionary differential forms are defined on manifolds with unclosed metric forms.

\section{Some properties of manifolds}

In the definition of exterior differential forms a differentiable manifold was mentioned. But differentiable manifolds are not a single type of manifolds on which the exterior differential forms are defined. In the general case there are manifolds with structures of any types. The theory of exterior differential forms was developed just for such manifolds. They may be the Hausdorff manifolds, fiber spaces, the comological, characteristical, configuration manifolds and so on. Since all these manifolds possess structures of any types, they have one common property, namely, locally they admit one-to-one mapping into Euclidean subspaces and into other manifolds or submanifolds of the same dimension [6].

When describing any processes in terms of differential equations, one has to deal with manifolds that do not allow one-to-one mapping described above. Such manifolds are, for example, manifolds formed by trajectories of elements of the system described by differential equations. The manifolds that can be called accompanying manifolds are variable deforming manifolds. Evolutionary differential forms can be defined on manifolds of this type.

The closed metric forms define the manifold structure, and the commutators of metric forms define the manifold differential characteristics that specify the manifold deformation: bending, torsion, rotation, twist. The topological properties of manifolds are connected with the metric form commutators. The metric form commutators specify a manifold distortion. For example, the commutator of the zero degree metric form $\Gamma_{\mu \nu}^{\rho}$ characterizes the bend, that of the first degree form $\left(\Gamma_{\mu \nu}^{\rho}-\Gamma_{\nu \mu}^{\rho}\right)$ characterizes the torsion, the commutator of the third-degree metric form $R_{\nu \rho \sigma}^{\mu}$ determines the curvature.

It is evident that the manifolds, that are metric ones or possess the structure, have closed metric forms. It is with such manifolds that the exterior differential forms are connected. 
If the manifolds are deforming manifolds, this means that their metric form commutators are nonzero. That is, the metric forms of such manifolds turn out to be unclosed. The accompanying manifolds and manifolds appearing to be deforming ones are examples of such manifolds.

The skew-symmetric evolutionary differential forms, whose basis are deforming manifolds, are defined on manifolds with unclosed metric forms.

Thus, the exterior differential forms are skew-symmetric differential forms defined on manifolds, submanifolds or on structures with closed metric forms. Evolutionary differential forms are skew-symmetric differential forms defined on manifolds with metric forms that are unclosed.

The evolutionary properties of the evolutionary skew-symmetric differential forms are just connected with properties of the metric form commutators.

\section{Specific features of the evolutionary differential form}

The evolutionary differential form of degree $p$ ( $p$-form) can be also written as an exterior differential form [2].

But the evolutionary form differential cannot be written similarly to that presented for exterior differential forms. In the evolutionary form differential there appears an additional term connected with the fact that the basis of the form changes [2].

For example, we again inspect the first-degree form $\omega=a_{\alpha} d x^{\alpha}$. [From here on the symbol $\sum$ will be omitted and it will be implied that a summation is performed over double indices. Besides, the symbol of exterior multiplication will be also omitted for the sake of presentation convenience].

The differential of this form can be written as $d \omega=K_{\alpha \beta} d x^{\alpha} d x^{\beta}$, where $K_{\alpha \beta}=a_{\beta ; \alpha}-a_{\alpha ; \beta}$ are components of the commutator of the form $\omega$, and $a_{\beta ; \alpha}, a_{\alpha ; \beta}$ are the covariant derivatives. If we express the covariant derivatives in terms of the connectedness (if it is possible), they can be written as $a_{\beta ; \alpha}=\partial a_{\beta} / \partial x^{\alpha}+\Gamma_{\beta \alpha}^{\sigma} a_{\sigma}$, where the first term results from differentiating the form coefficients, and the second term results from differentiating the basis. (In Euclidean space covariant derivatives coincide with ordinary ones since in this case derivatives of the basis vanish). If we substitute the expressions for covariant derivatives into the formula for the commutator components, we obtain the following expression for the commutator components of the form $\omega$ :

$$
K_{\alpha \beta}=\left(\frac{\partial a_{\beta}}{\partial x^{\alpha}}-\frac{\partial a_{\alpha}}{\partial x^{\beta}}\right)+\left(\Gamma_{\beta \alpha}^{\sigma}-\Gamma_{\alpha \beta}^{\sigma}\right) a_{\sigma}
$$

Here the expressions $\left(\Gamma_{\beta \alpha}^{\sigma}-\Gamma_{\alpha \beta}^{\sigma}\right)$ entered into the second term are just the components of commutator of the first-degree metric form.

The evolutionary form commutator of any degree contains the commutator of the manifold metric form of corresponding degree. The commutator of the exterior form does not contains a similar term because the commutator of metric form of manifold, on which the exterior form is defined, is equal to zero. 


\section{Unclosure of evolutionary differential forms}

The evolutionary differential form commutator, in contrast to that of the exterior one, cannot be equal to zero because it includes the metric form commutator being nonzero. This means that the evolutionary form differential is nonzero. Hence, the evolutionary differential form, in contrast to the case of the exterior form, cannot be closed.

The commutators of evolutionary forms depend not only on the evolutionary form coefficients, but also on the characteristics of manifolds, on which this form is defined. As a result, such a dependence of the evolutionary form commutator produces the topological and evolutionary properties of both the commutator and the evolutionary form itself (this will be demonstrated below).

Since the evolutionary differential forms are unclosed, the mathematical apparatus of evolutionary differential forms does not seem to possess any possibilities connected with invariant properties of closed exterior differential forms. However, the mathematical apparatus of evolutionary forms includes some new unconventional elements like nonidentical relations and degenerate transformations [3]. Just such peculiarities allow to describe evolutionary processes.

\section{Nonidentical relations of evolutionary differential forms and their specific features}

The identical relations of closed exterior differential forms reflect a conjugacy of any objects. The evolutionary forms, being unclosed, cannot directly describe a conjugacy of any objects. But they allow a description of the process in which the conjugacy may appear (a process when closed exterior differential forms are generated). Such a process is described by nonidentical relations.

Nonidentical relations can be written as

$$
d \psi=\omega^{p}
$$

Here $\omega^{p}$ is the $p$-degree evolutionary form that is nonintegrable, $\psi$ is some form of degree $(p-1)$, and the differential $d \psi$ is a closed form of degree $p$.

In the left-hand side of this relation it stands the form differential, i.e. a closed form that is an invariant object. In the right-hand side it stands the nonintegrable unclosed form that is not an invariant object. Such a relation cannot be identical.

One can see a difference of relations for exterior forms and evolutionary ones. In the right-hand side of identical relation (see relation (8)) it stands a closed form, whereas the form in the right-hand side of nonidentical relation (10) is an unclosed one.

Nonidentical relations are obtained while describing any processes. A relation of such type is obtained while analyzing the integrability of the partial differential equation. An equation is integrable if it can be reduced to the form 
$d \psi=d U$. However it appears that, if the equation is not subject to an additional condition (the integrability condition), it is reduced to the form (10), where $\omega$ is an unclosed form and it cannot be expressed as a differential. The first principle of thermodynamics is an example of nonidentical relation.

While investigating real physical processes one often faces the relations that are nonidentical. But it is commonly believed that only identical relations can have any physical and mathematical meaning. For this reason one immediately attempts to impose a condition onto the nonidentical relation under which this relation becomes identical, and it is considered only a relation that can satisfy the additional conditions. And all remaining is rejected.

In this approach the identical relations, which can be obtained from a given nonidentical relation, are obtained. So, the closed exterior forms and relevant differential-geometrical structures are found. However, this approach does not solve the evolutionary problem. In this approach there is no answer to the questions of how do the closed exterior differential forms appear, how do the structures originate. That is, in such approach there is no answer to the question of how the conditions, under which the closed exterior forms are obtained, are realized. It turns out that these conditions are realized while varying the nonidentical relation, which is a selfvarying relation.

\section{Selfvariation of the evolutionary nonidentical relation}

The evolutionary nonidentical relation is selfvarying, because, firstly, it is nonidentical, namely, it contains two objects one of which appears to be unmeasurable, and, secondly, it is an evolutionary relation, namely, a variation of any object of the relation in some process leads to variation of another object and, in turn, a variation of the latter leads to variation of the former. Since one of the objects is an unmeasurable quantity, the other cannot be compared with the first one, and hence, the process of mutual variation cannot terminate.

Varying the evolutionary form coefficients leads to varying the first term of the evolutionary form commutator (see (2.3)). In accordance with this variation it varies the second term, that is, the metric form of manifold varies. Since the metric form commutators specifies the manifold differential characteristics, which are connected with the manifold deformation (as it has been pointed out, the commutator of the zero degree metric form specifies the bend, that of second degree specifies various types of rotation, that of the third degree specifies the curvature), this points to the manifold deformation. This means that it varies the evolutionary form basis. In turn, this leads to variation of the evolutionary form, and the process of intervariation of the evolutionary form and the basis is repeated. Processes of variation of the evolutionary form and the basis are controlled by the evolutionary form commutator and it is realized according to the evolutionary relation.

The process of the evolutionary relation selfvariation plays a governing role in description of the evolutionary processes. 
The significance of the evolutionary relation selfvariation consists in the fact that in such a process it can be realized conditions under which the identical relation is obtained from the nonidentical relation. These are conditions of degenerate transformation.

\section{Degenerate transformations. Origination of differential- geometrical structures}

To obtain the identical relation from the evolutionary nonidentical relation, it is necessary that a closed exterior differential form should be derived from the evolutionary differential form, which is included into evolutionary relation. However, as it has been shown above, the evolutionary form cannot be a closed form. For this reason a transition from the evolutionary form is possible only to an inexact closed exterior form, which is defined on pseudostructure.

To the pseudostructure it is assigned a closed dual form (whose differential vanishes). For this reason a transition from the evolutionary form to a closed inexact exterior form proceeds only when the conditions of vanishing the dual form differential are realized, in other words, when the metric form differential or commutator becomes equal to zero.

Since the evolutionary form differential is nonzero, whereas the closed exterior form differential is zero, the transition from the evolutionary form to the closed exterior form is allowed only under degenerate transformation. The conditions of vanishing the dual form differential (the additional condition) are the conditions of degenerate transformation.

Such conditions can just be realized under selfvariation of the nonidentical evolutionary relation.

As it has been already mentioned, the evolutionary differential form $\omega^{p}$, involved into nonidentical relation (10) is an unclosed one. The commutator, and hence the differential, of this form is nonzero. That is,

$$
d \omega^{p} \neq 0
$$

If the conditions of degenerate transformation are realized, then from the unclosed evolutionary form one can obtain a differential form closed on pseudostructure. The differential of this form equals zero. That is, it is realized the transition

$d \omega^{p} \neq 0 \rightarrow$ (degenerate transformation) $\rightarrow d_{\pi} \omega^{p}=0, d_{\pi}{ }^{*} \omega^{p}=0$

As conditions of degenerate transformation (additional conditions) it can serve any symmetries of the evolutionary form coefficients or its commutator. (While describing material system such additional conditions are related, for example, to degrees of freedom of the material system).

Mathematically to the conditions of degenerate transformation there corresponds a requirement that some functional expressions become equal to zero. Such functional expressions are Jacobians, determinants, the Poisson brackets, residues, and others. 
Thus, while selfvariation of the evolutionary nonidentical relation the dual form commutator can vanish (due to the symmetries of the evolutionary form coefficients or its commutator). This means that it is formed the pseudostructure on which the differential form turns out to be closed. The emergence of the form being closed on pseudostructure points out to origination of the differential-geometrical structures.

\section{Obtaining identical relation from nonidentical one}

On the pseudostructure $\pi$ evolutionary relation (10) transforms into the relation

$$
d_{\pi} \psi=\omega_{\pi}^{p}
$$

which proves to be an identical relation. Indeed, since the form $\omega_{\pi}^{p}$ is a closed one, on the pseudostructure this form turns out to be a differential of some differential form. In other words, this form can be written as $\omega_{\pi}^{p}=d_{\pi} \theta$. Relation (12) is now written as

$$
d_{\pi} \psi=d_{\pi} \theta
$$

There are differentials in the left-hand and right-hand sides of this relation. This means that the relation is an identical one.

From evolutionary relation (10) it is obtained the identical on the pseudostructure relation. In this case the evolutionary relation itself remains to be nonidentical one. (At this point it should be emphasized that differential, which equals zero, is an interior one. The evolutionary form commutator becomes zero only on the pseudostructure. The total evolutionary form commutator is nonzero. That is, under degenerate transformation the evolutionary form differential vanishes only on pseudostructure. The total differential of the evolutionary form is nonzero. The evolutionary form remains to be unclosed.)

It can be shown that all identical relations of the exterior differential form theory are obtained from nonidentical relations (that contain the evolutionary forms) by applying degenerate transformations.

The degenerate transform is realized as a transition to nonequivalent coordinate system: a transition from the accompanying noninertial coordinate system to the locally inertial that. Evolutionary relation (10) and condition (11) relate to the system being tied to the accompanying manifold, whereas identical relations (12) may relate only to the locally inertial coordinate system being tied to a pseudostructure.

Transition from nonidentical relation (10) to identical relation (12) means the following. Firstly, it is from such a relation that one can obtain the differential $d_{\pi} \psi$ and find the desired function $\psi_{\pi}$ (a potential). And, secondly, an emergence of the closed (on pseudostructure) inexact exterior form $\omega_{\pi}^{p}$ (right-hand side of relation (12)) points to an origination of the conservative object. This object is a conservative quantity (the closed exterior form $\omega_{\pi}^{p}$ ) on the pseudostructure (the 
dual form ${ }^{*} \omega^{p}$, which defines the pseudostructure). This object is an example of the differential-geometrical structure (Bi-Structure).

This complex is a new conjugated object. Below it will be shown a relation between characteristics of these objects (the differential-geometrical structure) and characteristics of the evolutionary differential forms.

Thus, the mathematical apparatus of evolutionary differential forms describes a process of generation of the closed inexact exterior differential forms, and this discloses a process of origination of the differential-geometrical structure, namely, a new conjugated object.

It can be seen that the process of conjugating the objects and obtaining differential-geometrical structures is a mutual exchange between the quantities of different nature (for example, between the algebraic and geometric quantities, between the physical and spatial quantities) and vanishing some functional expressions (Jacobians, determinants and so on). This follows from the fact that a selfvariation of the nonidentical evolutionary relation and a transition from the nonidentical evolutionary relation to identical one develop as a result of mutual variations of the evolutionary form coefficients (which have the algebraic nature) and the manifold characteristics (which have the geometric nature), and a realization of the degenerate transformation with obeying additional conditions.

The evolutionary differential form is an unclosed form, that is, it is the form whose differential is not equal to zero. The differential of the exterior differential form equals zero. To the closed exterior form there correspond conjugated operators, whereas to the evolutionary form there correspond nonconjugated operators. A transition from the evolutionary form to the closed exterior form and origination of the differential-geometrical structures is a transition from nonconjugated operators to conjugated ones. This is expressed mathematically as a transition from a nonzero differential (the evolutionary form differential is nonzero) to a differential that equals zero (the closed exterior form differential equals zero).

\section{Characteristics of Bi-Structure}

Since the closed exterior differential form, which corresponds to the Bi-Structure arisen, was obtained from the nonidentical relation that involves the evolutionary form, it is evident that the Bi-Structure characteristics must be connected with those of the evolutionary form and of the manifold on which this form is defined, with the conditions of degenerate transformation and with the values of commutators of the evolutionary form and the manifold metric form.

The conditions of degenerate transformation, as it was said before, determine the pseudostructures. The first term of the evolutionary form commutator determines the value of the discrete change (the quantum), which the quantity conserved on the pseudostructure undergoes when transition from one pseudostructure to another. The second term of the evolutionary form commutator specifies a characteristics that fixes the character of the initial manifold de- 
formation, which took place before the Bi-Structure arose. (Spin is such an example).

A discrete (quantum) change of a quantity proceeds in the direction that is normal (more exactly, transverse) to the pseudostructure. Jumps of the derivatives normal to the potential surfaces are examples of such changes.

Bi-Structure may carry a physical meaning. Such binary objects are the physical structures from which the physical fields are formed. This has been shown by the author in the works [1,9].

The connection of Bi-Structure with the skew-symmetric differential forms allows to introduce a classification of Bi-structure in dependence on parameters that specify the skew-symmetric differential forms and enter into nonidentical and identical relation of the skew-symmetric differential forms. To determine these parameters one has to consider the problem of integration of the nonidentical evolutionary relation.

Under degenerate transformation from the nonidentical evolutionary relation one obtains a relation being identical on pseudostructure. Since the right-hand side of such a relation can be expressed in terms of differential (as well as the left-hand side), one obtains a relation that can be integrated, and as a result he obtains a relation with the differential forms of less by one degree.

The relation obtained after integration proves to be nonidentical as well.

The resulting nonidentical relation of degree $(p-1)$ (relation that includes the forms of the degree $(p-1)$ ) can be integrated once again if the corresponding degenerate transformation has been realized and the identical relation has been formed.

By sequential integrating the evolutionary relation of degree $p$ (in the case of realization of the corresponding degenerate transformations and forming the identical relation), one can get closed (on the pseudostructure) exterior forms of degree $k$, where $k$ ranges from $p$ to 0 .

In this case one can see that under such integration the closed (on the pseudostructure) exterior forms, which depend on two parameters, are obtained. These parameters are the degree of evolutionary form $p$ (in the evolutionary relation) and the degree of created closed forms $k$.

In addition to these parameters, another parameter appears, namely, the dimension of space. If the evolutionary relation generates the closed forms of degrees $k=p, k=p-1, \ldots, k=0$, to them there correspond the pseudostructures of dimensions $(N-k)$, where $N$ is the space dimension. \{It is known that to the closed exterior differential forms of degree $k$ there correspond skew-symmetric tensors of rank $k$ and to corresponding dual forms there do the pseudotensors of rank $(N-k)$, where $N$ is the space dimensionality. The pseudostructures correspond to such tensors, but only on the space formed.\} 


\section{The properties of pseudostructures and closed exterior forms. Forming fields and manifolds}

As mentioned before, the additional conditions, namely, the conditions of degenerate transformation, specify the pseudostructure. But at every stage of the evolutionary process it is realized only one element of pseudostructure, namely, a certain minipseudostructure. The additional conditions determine a direction (a derivative of the function that specifies the pseudostructure) on which the evolutionary form differential vanishes. (However, in this case the total differential of the evolutionary form is nonzero). The closed exterior form is formed along this direction.

While varying the evolutionary variable the minipseudostructures form the pseudostructure. The example of minipseudoctructure is the wave front. The wave front is an eikonal surface (the level surface), i.e. the surface with a conservative quantity. A direction that specifies the pseudostructure is a connection between the evolutionary and spatial variables. It gives the rate of changing the spatial variables. Such a rate is a velocity of the wave front translation. While its translation the wave front forms the pseudostructure.

Manifolds with closed metric forms are formed by pseudostructures. They are obtained from manifolds with unclosed metric forms. In this case the initial manifold (on which the evolutionary form is defined) and the manifold with closed metric forms originated (on which the closed exterior form is defined) are different spatial objects.

It takes place a transition from the initial manifold with unclosed metric form to the pseudostructure, namely, to the manifold with closed metric forms being created. Mathematically this transition (degenerate transformation) proceeds as a transition from one frame of reference to another, nonequivalent, frame of reference.

The pseudostructures, on which the closed inexact forms are defined, form the pseudomanifolds. (Integral surfaces, pseudo-Riemann and pseudo-Euclidean spaces are the examples of such manifolds). In this process dimensions of the manifolds formed are connected with the evolutionary form degree.

To transition from pseudomanifolds to metric manifolds it is assigned a transition from closed inexact differential forms to exact exterior differential forms. (Euclidean and Riemann spaces are examples of metric manifolds).

Here it is to be noted that the examples of pseudometric spaces are potential surfaces (surfaces of a simple layer, a double layer and so on). In these cases the type of potential surfaces is connected with the above listed parameters.

Since the closed metric form is dual with respect to some closed exterior differential form, the metric forms cannot become closed by themselves, independent of the exterior differential form. This proves that manifolds with closed metric forms are connected with the closed exterior differential forms. This indicates that the fields of conservative quantities are formed from closed exterior forms at the same time when the manifolds are created from the pseudoctruc- 
tures. (The specific feature of the manifolds with closed metric forms that have been formed is that they can carry some information.) That is, the closed exterior differential forms and manifolds, on which they are defined, are mutually connected objects. On the one hand, this shows a duality of these two objects

(the pseudostructure and the closed inexact exterior form), and, on the other hand, this means that these objects constitute a unified whole. This whole is a new conjugated object (Bi-Structure).

\section{References}

1. Petrova L. I., Exterior and evolutionary skew-symmetric differential forms and their role in mathematical physics.

http://arXiv.org/pdf/math-ph/0310050

2. Petrova L. I., Invariant and evolutionary properties of the skew-symmetric differential forms.

http://arXiv.org/pdf/math.GM/0401039

3. Petrova L. I., Identical and nonidentical relations. Nondegenerate and degenerate transformations. (Properties of the skew-symmetric differential forms). http://arXiv.org/pdf/math.GM/0404109

4. Cartan E., Les Systemes Differentials Exterieus ef Leurs Application Geometriques. -Paris, Hermann, 1945.

5. Bott R., Tu L. W., Differential Forms in Algebraic Topology. Springer, NY, 1982.

6. Schutz B. F., Geometrical Methods of Mathematical Physics. Cambridge University Press, Cambridge, 1982.

7. Encyclopedia of Mathematics. -Moscow, Sov. Encyc., 1979 (in Russian).

8. Wheeler J. A., Neutrino, Gravitation and Geometry. Bologna, 1960.

9. Petrova L. I., Conservation laws. Their role in evolutionary processes. (The method of skew-symmetric differential forms).

http://arXiv.org/pdf/math-ph/0311008 